\title{
Crystal Growth and Stoichiometry of Strongly Correlated Intermetallic Cerium Compounds
}

\author{
Andrey Prokofiev and Silke Paschen \\ Institute of Solid State Physics, Vienna University of Technology, \\ Wiedner Hauptstr. 8-10, 1040 Vienna \\ Austria
}

\section{Introduction}

Strongly correlated electron systems are among the most active research topics in modern condensed matter physics. In strongly correlated materials the electron interaction energies dominate the electron kinetic energy which leads to unconventional properties. Heavy fermion compounds form one of the classes of such materials. In heavy fermion compounds the interaction of itinerant electrons with local magnetic moments generates quasiparticles with masses up to several 1000 electron masses. This may be accompanied by exciting properties, such as unconventional superconductivity in a magnetic environment, non-Fermi liquid behavior and quantum criticality. Strong electronic correlations are responsible for physical phenomena on a low energy scale. Consequently, these phenomena have to be studied at low temperatures. This, in turn, requires ultimate quality of single crystals to avoid that the low temperature intrinsic properties are covered by extrinsic effects due to off-stoichiometry, impurities or other crystal imperfections.

The overwhelming majority of heavy fermion systems are cerium and ytterbium intermetallic compounds. In the present paper we discuss the crystal growth of three cerium compounds, $\mathrm{Ce}_{3} \mathrm{Pd}_{20} \mathrm{Si}_{6}, \mathrm{CeRu}_{4} \mathrm{Sn}_{6}$ and $\mathrm{CeAuGe}$. $\mathrm{Ce}_{3} \mathrm{Pd}_{20} \mathrm{Si}_{6}$ undergoes an antiferromagnetic phase transition at low temperatures and shows a magnetic field induced quantum critical point [Takeda et al (1995), Strydom et al (2006)]. CeRu $\mathrm{Sn}_{6}$ [Das \& Sampathkumaran (1992)] appears to be a Kondo insulator [Aepli \& Fisk (1992)] with a highly anisotropic energy gap. $\mathrm{CeAuGe}$ is one of a the few cerium compounds showing a ferromagnetic phase transition at low temperatures [Pöttgen et al (1998), Mhlungu \& Strydom (2008)]. This is of special interest in the context of quantum criticality, since the occurrence of quantum criticality on the verge of a ferromagnetic ground state is a subject of current debate.

Much attention in this paper is paid to the problem of stoichiometry. Single crystals of intermetallic compounds are grown at high temperatures, which facilitates the formation of thermal defects realized often as deviation from the stoichiometric composition. Thermal instabilities of some intermetallic phases require the use of flux techniques, i.e., growth from off-stoichiometric melts, which is another source of non-stoichiometry.

Sizeable non-stoichiometries can be detected by measuring the composition by chemical and physical analytical techniques, e.g. energy dispersive X-ray spectroscopy analysis (EDX). Tiny deviations from the stoichiometry, on the other hand, can be found only from an analysis of the physical properties of single crystals. Thus physical property measurements are not only the 
final purpose of a crystal growth but also a valuable diagnostic tool for further improvement of their quality. Therefore, in the paper the consideration of crystal growth is accompanied by the discussion of their physical properties.

Physical property measurements on $\mathrm{Ce}_{3} \mathrm{Pd}_{20} \mathrm{Si}_{6}$ single crystals grown by the floating zone technique have been reported earlier [Goto et al (2009), Prokofiev et al (2009), Mitamura et al (2010)]. In Ref. Prokofiev et al (2009) a systematic study of the relationship between the growth technique, stoichiometry and physical properties of single crystals has been done. The crystal growth and stoichiometry of $\mathrm{CeRu}_{4} \mathrm{Sn}_{6}$ and CeAuGe are reported for the first time. The physical properties of the $\mathrm{CeRu}_{4} \mathrm{Sn}_{6}$ single crystals were published partially in an author's earlier paper [Paschen et al (2010)].

\section{2. $\mathrm{Ce}_{3} \mathrm{Pd}_{20} \mathrm{Si}_{6}$}

The recent observation of magnetic-field induced quantum criticality [Paschen et al (2007); Strydom et al (2006)] in the cubic heavy fermion compound $\mathrm{Ce}_{3} \mathrm{Pd}_{20} \mathrm{Si}_{6}$ [Takeda et al (1995)] has attracted considerable attention. $\mathrm{Ce}_{3} \mathrm{Pd}_{20} \mathrm{Si}_{6}$ crystallizes in a cubic $\mathrm{Cr}_{23} \mathrm{C}_{6}$-type structure with the space group $F m \overline{3} m$ [Gribanov et al (1994)]. The cubic cell with $a=12.161 \AA$ [Gribanov et al (1994)]; 12.280 ̊ [Takeda et al (1995)] contains 116 atoms. The Ce atoms occupy two distinct sites of cubic point symmetry. At the octahedral $4 a$ (tetrahedral $8 c$ ) site $\mathrm{Ce}$ atoms are situated in cages formed by $12 \mathrm{Pd}$ and $6 \mathrm{Si}$ atoms (16 $\mathrm{Pd}$ atoms). These high coordination numbers allow to classify $\mathrm{Ce}_{3} \mathrm{Pd}_{20} \mathrm{Si}_{6}$ as a cage compound. Strongly correlated cage compounds are of much interest as potential candidates for thermoelectric applications [Paschen (2006)].

Similar to the isostructural germanide compound $\mathrm{Ce}_{3} \mathrm{Pd}_{20} \mathrm{Ge}_{6}$, two phase transitions - a presumably antiferromagnetic one at $T_{L}$ of $0.15 \mathrm{~K}$ [Takeda et al (1995)], 0.17 K [Goto et al (2009)], or $0.31 \mathrm{~K}$ [Strydom et al (2006)] and a possibly quadrupolar one at $T_{U}$ of $0.5 \mathrm{~K}$ [Strydom et al (2006)] - have been found in the silicide compound $\mathrm{Ce}_{3} \mathrm{Pd}_{20} \mathrm{Si}_{6}$. Similar to the effect of magnetic field applied along [100] or [110] in $\mathrm{Ce}_{3} \mathrm{Pd}_{20} \mathrm{Ge}_{6}$ [Kitagawa (1998)], a magnetic field shifts the two transitions of polycrystalline $\mathrm{Ce}_{3} \mathrm{Pd}_{20} \mathrm{Si}_{6}$ in opposite directions: At the critical field of about $1 \mathrm{~T}$ the transition at $T_{L}$ is suppressed to zero whereas the transition at $T_{U}$ shifts to $0.67 \mathrm{~K}$ [Strydom et al (2006)]. The non-Fermi liquid behavior of the electrical resistivity observed at the critical field in the temperature range $0.1-0.6 \mathrm{~K}$ is an indication for the existence of a field-induced quantum critical point [Paschen et al (2007)].

Neutron scattering experiments on polycrystalline $\mathrm{Ce}_{3} \mathrm{Pd}_{20} \mathrm{Si}_{6}$ have to date failed to detect any kind of magnetic order [Paschen et al (2008)]. Thus, to clarify the nature of the phases below $T_{L}$ and $T_{U}$ large single crystals of high quality are needed. In fact, in the first neutron scattering study on $\mathrm{Ce}_{3} \mathrm{Pd}_{20} \mathrm{Si}_{6}$ single crystals [Paschen et al (2008)] the absence of signatures of magnetic order was attributed to a too low $T_{L}$ value of the investigated specimen.

Since both phase transitions take place at rather low temperatures, disorder may influence them significantly. The discrepancy in the reported ordering temperatures (e.g. [Goto et al (2009); Strydom et al (2006); Takeda et al (1995)]) demonstrates this delicate dependence. Also the non-negligible difference in the reported unit cell constants [Gribanov et al (1994), Takeda et al (1995)] needs a clarification. This has motivated us to undertake a systematic investigation of the relation between crystal growth techniques/conditions, sample quality, and the resulting physical properties down to dilution refrigerator temperatures.

We show here that both phase transitions are extremely sensitive to small stoichiometry variations that result from different growth procedures. 
To elucidate the discrepancies in the low-temperature data reported on the quantum critical heavy fermion compound $\mathrm{Ce}_{3} \mathrm{Pd}_{20} \mathrm{Si}_{6}$ and reveal the compound's intrinsic properties, single crystals of varying stoichiometry were grown by various techniques - from the stoichiometric and slightly off-stoichiometric melts as well as from high temperature solutions using fluxes of various compositions. The results of this work on $\mathrm{Ce}_{3} \mathrm{Pd}_{20} \mathrm{Si}_{6}$ have been partially reported earlier [Prokofiev et al (2009)]. Here a more detailed analysis including also information on new crystal growth experiments as well as the physical property measurements on new single crystals are reported.

\subsection{Growth from the stoichiometric melt}

To investigate the melting character of $\mathrm{Ce}_{3} \mathrm{Pd}_{20} \mathrm{Si}_{6}$ a differential thermal analysis (DTA) run up to $1350^{\circ} \mathrm{C}$ was carried out (Fig. 1). There is only a single peak both on the heating and on the cooling curve, indicating congruent melting as previously reported [Gribanov et al (1994)]. A closer inspection of the shape of the melting peak (Fig.1, inset) might, however, suggest merely a quasi-congruent melting character. The onset of melting occurs at $T_{M} \approx 1250^{\circ} \mathrm{C}$. Because of undercooling the crystallization begins about $100^{\circ} \mathrm{C}$ lower than the melting.

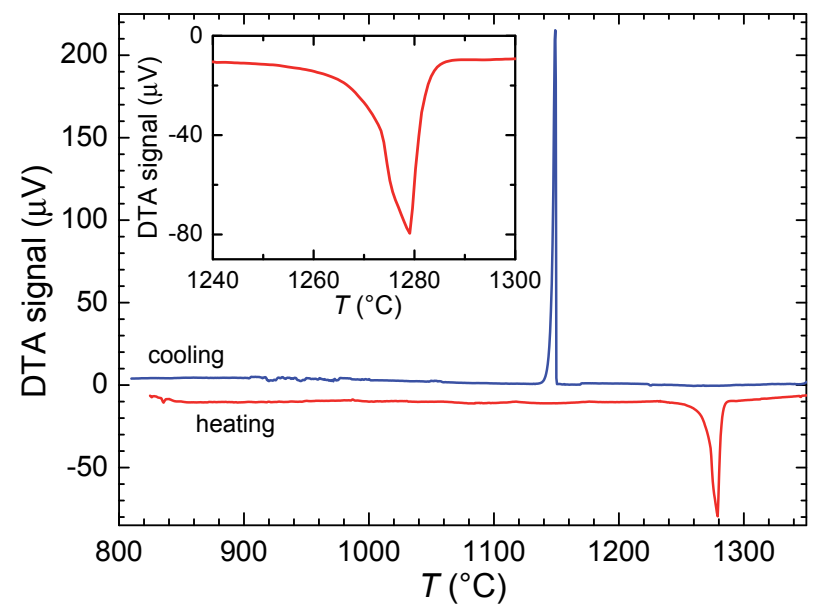

Fig. 1. DTA heating and cooling curves of $\mathrm{Ce}_{3} \mathrm{Pd}_{20} \mathrm{Si}_{6}$. The inset shows a magnification of the melting peak. From Ref. Prokofiev et al (2009).

The floating zone technique with optical heating was used for crystal growth from the melt, with a pulling rate of $10 \mathrm{~mm}$ per hour and an upper rod rotation speed of $5 \mathrm{rpm}$. Due to the high density and the low surface tension of the $\mathrm{Ce}_{3} \mathrm{Pd}_{20} \mathrm{Si}_{6}$ melt the floating zone was rather unstable, and the melt dropped down repeatedly (Fig. 2, right). However, one growth could be kept running long enough to grow an ingot of $25 \mathrm{~mm}$ length (Fig. 2, left).

Over the growth run the originally shiny and clear surface of the melt became more and more opaque, and a crust on the surface could be seen after some time. This is a sign of incongruent melting supposedly due to a peritectic reaction. The crust is the higher temperature phase, therefore it forms on the optically heated surface which is the hottest place of the melt. This observation can be explained by close proximity of the peritectic point to the temperature of the complete melting of the system with the total composition $\mathrm{Ce}_{3} \mathrm{Pd}_{20} \mathrm{Si}_{6}$, as indicated already by the peculiar shape of the DTA curve (Fig. 1 inset). Therefore the formation of 


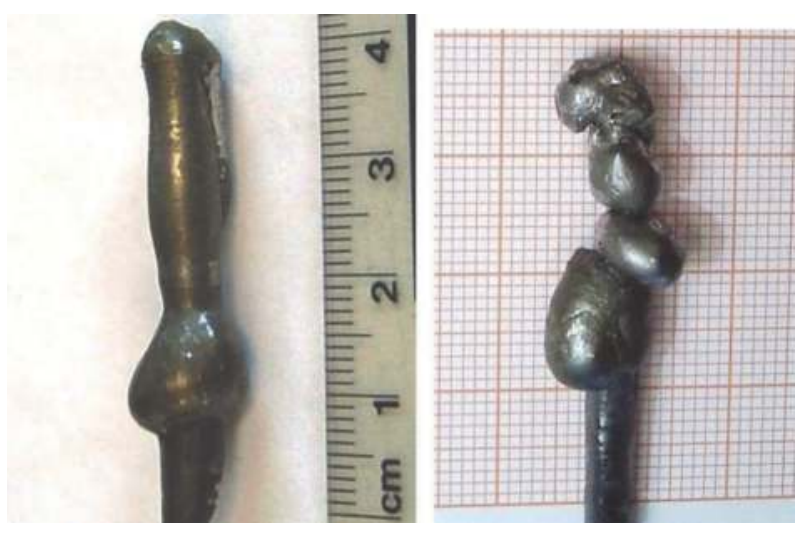

Fig. 2. Samples resulting from crystal growth experiments from the stoichiometric melt with upper and lower rod rotation. Left - Successfully grown single crystal (sc1); Right - The crystallized ingot experienced repeated dropping down of the melt because of the unfavorable combination of the high density and of the low surface tension of the melt.

a small amount of foreign phase on the hot surface results in a slight shift of the melt composition, which returns the crystallization process in the melt deep into the primary crystallization field of the $\mathrm{Ce}_{3} \mathrm{Pd}_{20} \mathrm{Si}_{6}$ phase.

Due to the thin foreign phase film the surface of the recrystallized (lower) rod was yellowish. The EDX measurement detected Ce ( 70 at.\%), Pd ( 22 at.\%), and $\mathrm{Si}(\sim 8$ at. $\%)$ in this film. However no inclusions of foreign phases were found by scanning electron microscopy (SEM) inside the crystal. A single crystal grown in this way is specified here as sc1 (see Table 1 for the nomenclature of all crystals). In order to trace segregation effects we differentiate additionally between the part of this crystal grown at the beginning of the growth process (bottom part of the ingot, $s c 1 b$ ) and that grown at the end (top part, sc1t). For the growth of $s c 1$ the lower purity starting materials (Ce 99.99 at.\%, Pd 99.95 at.\%) were used.

Further growth runs with rotation of the upper rod led to a permanent breaking of the surface crust. The melt leaked out through the cracks of the crust. Hence, the crust may serve as a quasi-crucible if it remains intact during the whole growth time. Based on this observation a growth run without rotation of the upper rod was carried out with the same growth parameters. The melt zone was quite stable in this experiment. A crystal grown by this procedure will be specified as $s c 2$. For the growth of this crystal higher purity starting materials (Ce 99.99 at.\%, Pd 99.998 at.\%) were used.

Laue investigations show very good crystallographic perfection of both types of crystals grown from the melt. Before annealing an SEM/EDX investigation of both crystals was carried out. The polished cross-section of the $s c 1$ rod is more homogeneous in composition than that of $s c 2$. The surface of the latter had a well distinguishable $300 \mu \mathrm{m}$ thick outer shell where the concentration of Ce was about $5 \%$ higher and that of Si somewhat lower than in the core region (Fig. 3, left). This shell may result from a partial dissolution and diffusion of the crust into the core of the rod. In the core region of $s c 2$ diffuse $10 \mu \mathrm{m}$ inclusions of supposedly the same shell phase, however with lower Ce concentration, were found (Fig. 3, right). These inclusions were not detected by X-ray powder diffraction and they disappeared after annealing, according to SEM analysis. 

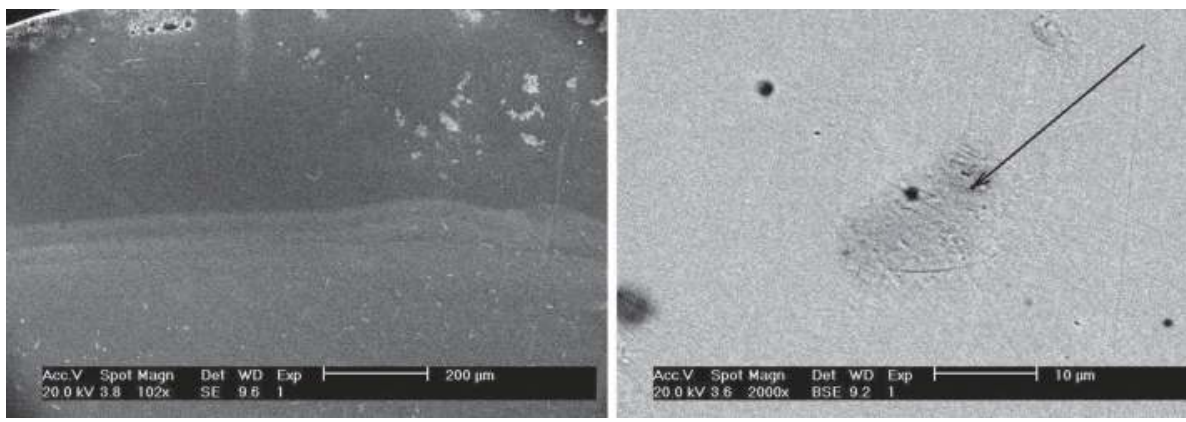

Fig. 3. Microstructure of $s c 2$ before annealing.

Left - Surface shell (darker top part) with a 5\% higher Ce concentration; Right - An inclusion with a lower Ce concentration in the core (shown by arrow).

After annealing of both crystals for 3 weeks at $900^{\circ} \mathrm{C}$ a second EDX investigation was carried out. At first we did the analysis without any reference sample. This yielded a stoichiometric Ce content (10.3 at.\%) but an over-stoichiometric Si content (22.0 instead of 20.7 at.\%) and an under-stoichiometric Pd content (67.7 instead of 69.0 at.\%) for both $s c 1$ and $s c 2$, corresponding to a partial substitution of Pd by Si on their sites. These results motivated our efforts to grow crystals using the flux technique, where the stoichiometry of the crystals can be tuned through the variation of the flux composition. The results of further EDX investigations with a reference sample will be discussed in Sect. 2.4.

\begin{tabular}{|l|l|}
\hline Sample name & Growth technique details \\
\hline \hline$s c 1 t$ & from melt with rod rotation, top part \\
\hline$s c 1 b$ & from melt with rod rotation, bottom part \\
\hline$s c 2$ & from melt without rod rotation \\
\hline$s c 3$ & $\begin{array}{l}\text { from } \mathrm{Pd}_{5} \mathrm{Si} \mathrm{flux}_{1} \\
\text { molar } \mathrm{Ce}_{3} \mathrm{Pd}_{20} \mathrm{Si}_{6} \text { to } \mathrm{Pd}_{5} \mathrm{Si} \text { ratio } 2: 1\end{array}$ \\
\hline sc4 & $\begin{array}{l}\text { from } \mathrm{Pd}_{5} \mathrm{Si}_{\text {flux }} \\
\text { molar } \mathrm{Ce}_{3} \mathrm{Pd}_{20} \mathrm{Si}_{6} \text { to } \mathrm{Pd}_{5} \text { Si ratio } 1: 2\end{array}$ \\
\hline$s c 5$ & $\begin{array}{l}\text { from slightly off-stoichiometric melt, } \\
\text { with a } 0.3 \text { at. } \% \text { Ce-excess and a } 0.2 \text { at.\% Pd-deficiency }\end{array}$ \\
\hline Sn flux crystals & using Sn or Sn-Pd fluxes \\
\hline
\end{tabular}

Table 1. Nomenclature of the $\mathrm{Ce}_{3} \mathrm{Pd}_{20} \mathrm{Si}_{6}$ crystals grown by various techniques.

\subsection{Flux growth}

\subsubsection{Tin flux}

We tried at first a crystal growth with standard fluxes. The related compound $\mathrm{Ce}_{2} \mathrm{Pd}_{3} \mathrm{Si}_{5}$ can be grown with $\mathrm{Sn}$ as flux at below $1100^{\circ} \mathrm{C}$ [Dung et al (2007)]. To check for the compatibility of Sn flux with $\mathrm{Ce}_{3} \mathrm{Pd}_{20} \mathrm{Si}_{6}$, a mixture of $\mathrm{Ce}_{3} \mathrm{Pd}_{20} \mathrm{Si}_{6}$ and $\mathrm{Sn}$ was heated up to $1100^{\circ} \mathrm{C}$ in a boron nitride crucible and then cooled slowly $\left(1^{\circ} \mathrm{C} / \mathrm{h}\right)$ down to $700^{\circ} \mathrm{C}$. The solute-to-solvent ratio was 2:1. After crystallization the ingot was cut, polished, and investigated by SEM/EDX and X-ray powder diffraction (XRD).

The crystallization yielded relatively large single crystals of the non-stoichiometric phase $\mathrm{CePd}_{2-x} \mathrm{Si}_{2+x}$ with $x \approx 0.25$, incorporated in a matrix of $\mathrm{Sn}_{4} \mathrm{Pd}$. In addition, small inclusions 
of other phases were found. The experiment thus shows the inapplicability of Sn as a flux because its affinity to $\mathrm{Pd}$ leads to a destruction of the $\mathrm{Ce}_{3} \mathrm{Pd}_{20} \mathrm{Si}_{6}$ phase.

However, other Sn-based flux compositions with a lower affinity to Pd can be found in the Sn-Pd binary phase diagram [Chandrasekharaiah (1990)]. A series of Sn-Pd compounds $\mathrm{Sn}_{4} \mathrm{Pd}, \mathrm{Sn}_{3} \mathrm{Pd}, \mathrm{Sn}_{2} \mathrm{Pd}$ - with low melting points (below $600^{\circ} \mathrm{C}$ ) exists.

Similar experiments as the one with pure $\mathrm{Sn}$ flux were carried out using the above Sn-Pd compositions. In all cases the primarily crystallized phase was $\mathrm{CePd}_{2-x} \mathrm{Si}_{2+x}$ according to SEM/EDX. The $x$-value diminished with increasing Pd content in the flux, reaching about 0.05 for $\mathrm{Sn}_{2} \mathrm{Pd}$ flux. The stoichiometric $\mathrm{CePd}_{2} \mathrm{Si}_{2}$ phase has the same $\mathrm{Ce} / \mathrm{Si}$ ratio as the $\mathrm{Ce}_{3} \mathrm{Pd}_{20} \mathrm{Si}_{6}$ phase but a strongly reduced $\mathrm{Pd}$ content. However, further increasing of the $\mathrm{Pd}$ concentration in the flux for tuning of Pd content in crystals was impossible: with the higher melting compound $\mathrm{SnPd}$, only partial melting of the crucible content was observed at $1100^{\circ} \mathrm{C}$. Thus, foreign flux growth was not successful.

\subsubsection{Self-flux $\mathrm{Pd}_{5} \mathrm{Si}$}

The reason for using the flux method was, on one hand, to obtain single crystals with exact stoichiometric composition and, on the other hand, the expectation that the off-stoichiometric melt would have a higher surface tension and hence the floating zone would be more stable than without flux. To avoid a contamination by foreign atoms we first opted for self flux growth. Since the concentration of thermal defects (e.g., $\mathrm{Si}$ - Pd substitutions) in the crystal is expected to decrease with decreasing growth temperature, we searched in the Ce-Pd-Si phase diagram (Fig. 4a) for low-melting (at first binary) compositions with an over-stoichiometric Pd content.

The phase $\mathrm{Pd}_{5} \mathrm{Si}$ [Seropegin (2001)] which, according to a later study [Gribanov et al (2006)], appears to consists or two scarcely distinguished phases $\mathrm{Pd}_{14} \mathrm{Si}_{3}$ and $\mathrm{Pd}_{84} \mathrm{Si}_{16}$, fulfills all requirements: it melts at a relatively low temperature of $835^{\circ} \mathrm{C}$ [Chandrasekharaiah (1990)], has an over-stoichiometric ( $>20: 6) \mathrm{Pd} / \mathrm{Si}$ ratio, and there are no stable Ce-containing intermediate phases between $\mathrm{Pd}_{5} \mathrm{Si}$ and $\mathrm{Ce}_{3} \mathrm{Pd}_{20} \mathrm{Si}_{6}$ in the Ce-Pd-Si ternary phase diagram (cross-section at $600^{\circ} \mathrm{C}$ [Gribanov et al (2006); Seropegin (2001)]).

As above, the floating zone technique with optical heating was used. The feed and the seed rods had the stoichiometric starting composition $\mathrm{Ce}_{3} \mathrm{Pd}_{20} \mathrm{Si}_{6}$, and the zone was a molten mixture of $\mathrm{Ce}_{3} \mathrm{Pd}_{20} \mathrm{Si}_{6}$ and $\mathrm{Pd}_{5} \mathrm{Si}$. Contrary to the melt growth, the floating zone was very stable, and its surface remained clear during the entire growth run. The latter means that the growth occurred within the $\mathrm{Ce}_{3} \mathrm{Pd}_{20} \mathrm{Si}_{6}$ primary crystallization field. The stability of the melt zone allowed the rotation of the upper rod. The pulling rate was $0.6 \mathrm{~mm} / \mathrm{h}$. Two growth runs with different flux compositions (molar ratios $\mathrm{Ce}_{3} \mathrm{Pd}_{20} \mathrm{Si}_{6}$ to $\mathrm{Pd}_{5} \mathrm{Si}$ of 2:1 and 1:2) were carried out (Fig. 4b). The crystals were annealed for 3 weeks at $900^{\circ} \mathrm{C}$. The corresponding samples are referred to as $s c 3$ and $s c 4$, respectively.

\subsection{Growth from slightly off-stoichiometric melt}

As the analysis of the composition and the properties of the crystals sc1-sc4 grown from the melt and from flux has shown (see Sections $2.4,2.5$ ) the stoichiometry was strongly sensitive to the starting composition of the melt. For a fine correction of non-stoichiometry a growth from a slightly off-stoichiometric melt was carried out, too (sc5). For reasons discussed later, the feed rod for sc5 contained 0.3 at.\% excess of Ce and 0.2 at.\% deficiency of Pd. To avoid the floating zone instability the growth was carried out without rod rotation, as in case of $s c 2$. 


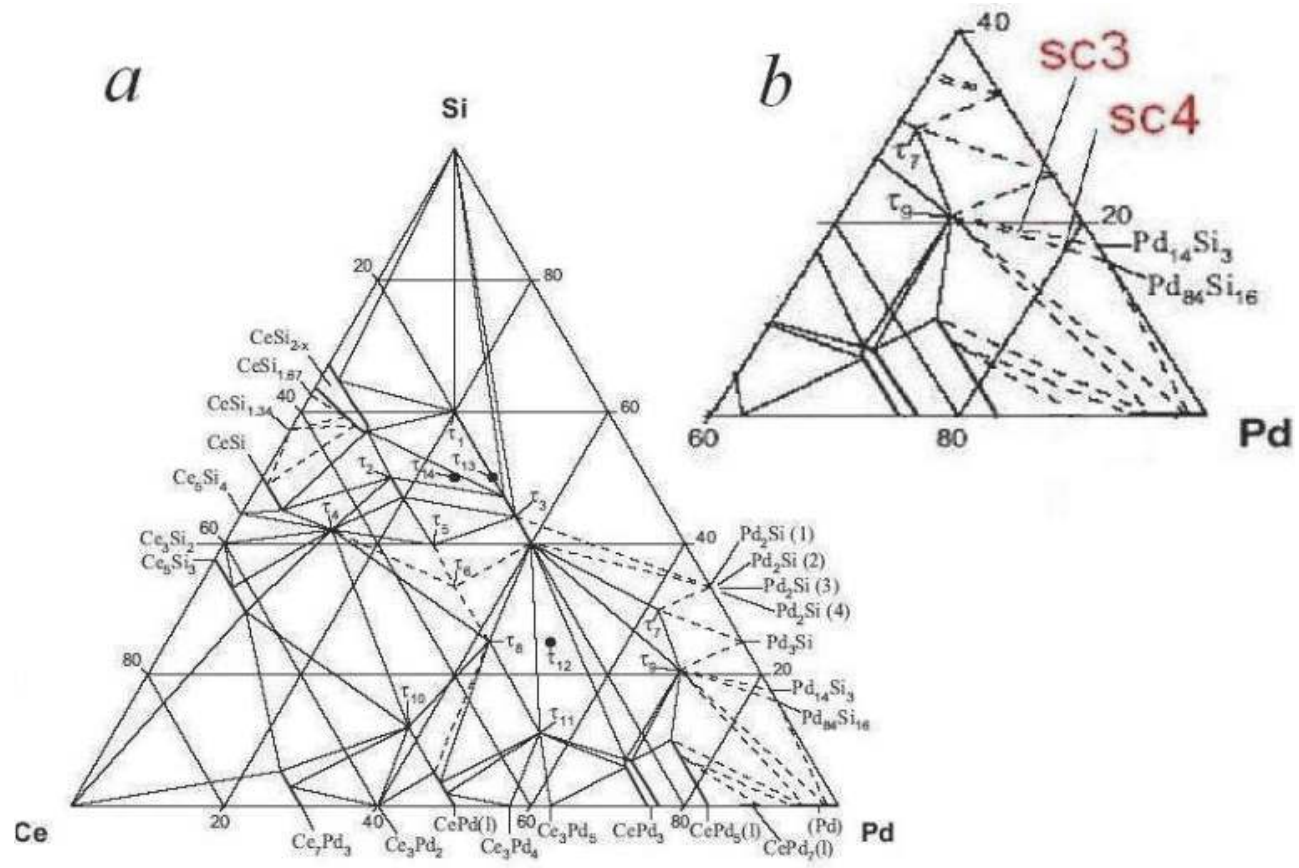

Fig. 4. a) Phase diagram of the Ce-Pd-Sn ternary system (from Gribanov et al (2006)). Here $\mathrm{Ce}_{3} \mathrm{Pd}_{20} \mathrm{Si}_{6}$ is denoted as $\tau_{9}$-phase. $b$ ) Magnified part of the phase diagram with the compositions of the solutions from that $s c 3$ and $s c 4$ were grown.

The pulling rate was $4 \mathrm{~mm} / \mathrm{h}$. Similar to $s c 1$ we differentiate additionally between the bottom and top parts of the ingot, $s c 5 b$ and $s c 5 t$.

\subsection{Composition of the grown crystals}

The usual EDX technique without standards cannot determine absolute atomic concentrations with sufficient accuracy. This problem can, in principle, be solved by using a standard of exactly known composition. However, as such a sample is not readily available, we used, instead, the polycrystalline sample $(p c)$ which will be shown below to be of best quality, according to the physical property measurements. Irrespective of whether its composition may be identified with the exact stoichiometry 3:20:6 or not it served as a practical guideline to establish the crystal composition-property relationship.

With additional improvements of our EDX setup (improved measurement statistics, counting time, beam current control) we can measure, with a high accuracy, deviations from the reference sample stoichiometry. The results are summarized in Table 2 where the measured lattice parameters are given, too. Because crystals grown from the Sn-containing fluxes are not the title phase and their compositions vary sizably they are simply represented by $\mathrm{CePd}_{2-x} \mathrm{Si}_{2+x}$ in Table 2.

Even though, in the absence of a real standard, there remains some uncertainty about the absolute values measured by EDX (which even for the reference sample $p c$ differ from the calculated stoichiometry 3:20:6), trends in the composition of the investigated series of crystals 


\begin{tabular}{|c|c|c|c|}
\hline \multirow[t]{2}{*}{ Sample name } & \multicolumn{2}{|c|}{ Composition (at.\%) } & \multirow[t]{2}{*}{$a(\AA)$} \\
\hline & Ce $\mathrm{Pd}$ & $\mathrm{Si}$ & \\
\hline$s c 1 t$ & 10.5 & 21.9 & 12.276 \\
\hline$s c 1 b$ & 67.7 & 22.4 & 12.272 \\
\hline$s c 2$ & 68.0 & 22.1 & 12.272 \\
\hline$s c 3$ & 68.7 & 22.1 & 12.233 \\
\hline sc4 & 70.1 & 22.5 & 12.180 \\
\hline sc5 & 10.7 & 21.3 & 12.277 \\
\hline$p c$ & 10.1 & 22.2 & 12.280 \\
\hline Exact 3:20:6 stoichiometry & $10.3 \quad 69.0$ & 20.7 & \\
\hline Sn flux crystals & $\mathrm{CePd}_{2-x}$ & $2+x$ & \\
\hline Standard deviation & $0.24 \quad 0.20$ & 0.20 & \\
\hline Systematic error & & & $<0.002$ \\
\hline
\end{tabular}

Table 2. Composition and lattice parameter $a$ of the investigated $\mathrm{Ce}_{3} \mathrm{Pd}_{20} \mathrm{Si}_{6}$ samples.

can be discussed. While the Si content varies only weakly a stronger variation of the Ce and Pd content is observed, the Ce concentration nearly anticorrelating with the one of Pd (Fig. 5).

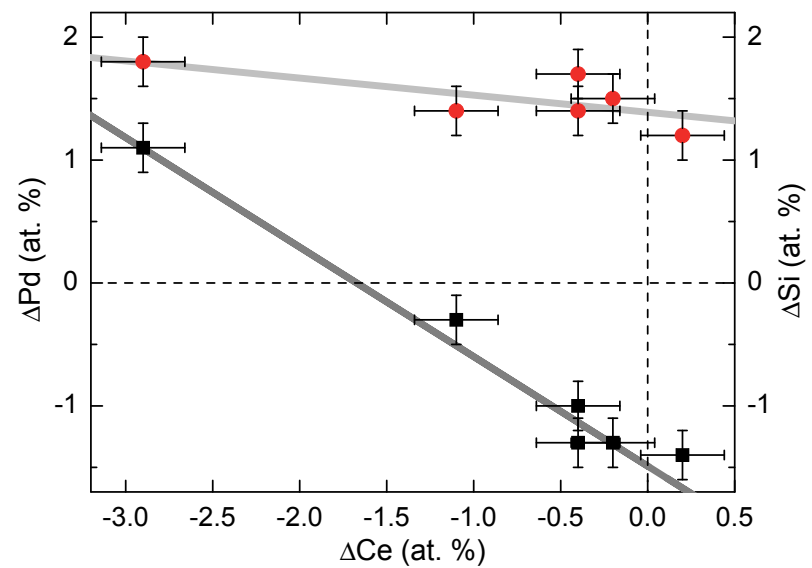

Fig. 5. Deviation of the Pd and Si content, $\Delta \mathrm{Pd}$ and $\Delta \mathrm{Si}$, from the exact 3:20:6 stoichiometry as function of the Ce non-stoichiometry $\Delta \mathrm{Ce}$. The dashed lines indicate the exact stoichiometry. The meaning of the broad grey lines is discussed in the text. Error bars indicate the standard deviations given in Table 2. From Ref. Prokofiev et al (2009).

Ce over-stoichiometry can be realized by a substitution of $\mathrm{Pd}$ or $\mathrm{Si}$ atoms on their crystallographic sites by excess Ce-atoms or, alternatively, by vacancies on the $\mathrm{Pd}$ and/or $\mathrm{Si}$ sites. Since the lattice parameter of $s c 1 t$ and $s c 5$ is smaller than that of the $p c$, the latter option is more probable, but a combination of both options cannot be excluded. If only $\mathrm{Pd} / \mathrm{Si}$ vacancies were present, the Ce sublattice would remain occupied and fully ordered. Ce under-stoichiometry, on the other hand, can be associated either with $\mathrm{Ce}$ vacancies or, which is more probable taking into account the approximate anti-correlation between the $\mathrm{Ce}$ and Pd contents, with a partial Pd substitution on Ce sites. The broad dark grey line in Fig. 5 represents the Ce-Pd concentration relation for a crystallochemical model which, as an example, assumes a half-filling of the Ce vacancies $V$ by excess $\mathrm{Pd}$ atoms: 
$\left[\mathrm{Ce}_{1-x} V_{0.5 x} \mathrm{Pd}_{0.5 x}\right]_{3} \mathrm{Pd}_{20} \mathrm{Si}_{6}$. The same model was used to describe the $\Delta \mathrm{Si}$ vs $\Delta$ Ce relation (light grey line). The agreement with the data is excellent.

\subsection{Influence of the growth technique on the physical properties}

The resulting stoichiometry of the crystals as well as their physical properties show sizable dependence on the different growth techniques.

Neutron diffraction experiments were carried out on an oriented sample cut from $s c 1 b$. The crystal was confirmed to have excellent crystallinity but, contrary to our expectations from the investigations on polycrystalline samples, showed no phase transition down to the lowest accessed temperature of about $0.15 \mathrm{~K}$ [Paschen et al (2008)]. The present investigation shows that, for this very single crystal, this temperature was still too high.

The specific heat $C_{p}$ was measured for $s c 1-s c 3$ and $s c 5$. Figure 6a shows the temperature dependence of $C_{p} / T$ of these crystals, together with published data of a polycrystalline sample [Strydom et al (2006)]. $C_{p} / T(T)$ of the polycrystalline sample $p c$ has a sharp peak with a maximum at $T_{L}=0.31 \mathrm{~K}$ and a shoulder at $T_{U} \sim 0.5 \mathrm{~K}$ [Strydom et al (2006)]. These features get successively broadened and suppressed to lower temperatures in the crystals $s c 1 t$, $s c 5 b$, $s c 1 b$, and $s c 2$, respectively. In $s c 3$ no clear signature of $T_{L}$ can be identified, suggesting that it has shifted further to lower temperatures and was further broadened or, alternatively, has vanished altogether. Due to the suppression of this lower temperature feature the anomaly at $T_{U}$, identified in all other samples as shoulder, now appears as a maximum.

From the specific heat measurements the best single crystals thus appear to be $s c 1 t$ and $s c 5$. With their rather sharp peaks at $0.22 \mathrm{~K}$ and $0.20 \mathrm{~K}$, respectively, and a shoulder at about $0.4 \mathrm{~K}$ they demonstrate all features associated with the intrinsic behavior of $\mathrm{Ce}_{3} \mathrm{Pd}_{20} \mathrm{Si}_{6}$.

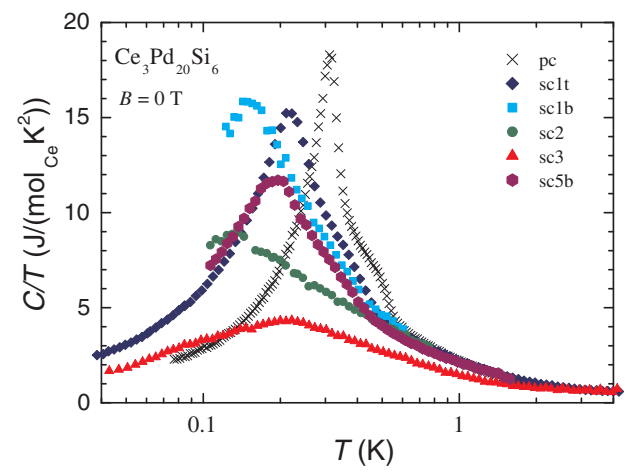

(a)

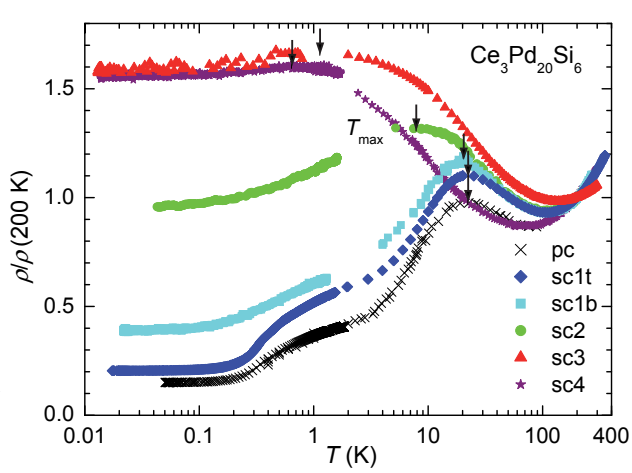

(b)

Fig. 6. a) Specific heat divided by temperature $C_{p} / T$ plotted for all single crystals prepared here, and for a polycrystal [Strydom et al (2006)] for comparison, as function of temperature $T$ on a logarithmic scale. The lower transition temperatures $T_{L}$ are taken here as position of the maxima. The maximum in the data for $s c 3$ can be identified with $T_{U}$ (see text).

b) Electrical resistivity of the $\mathrm{Ce}_{3} \mathrm{Pd}_{20} \mathrm{Si}_{6}$ single crystals grown here normalized to the respective values $\rho_{200} \mathrm{~K}$ vs temperature $T$. The arrows indicate the positions $T_{\max }$ of the (local) maxima. Data of a polycrystal [Strydom et al (2006)] are shown for comparison. From Ref. Prokofiev et al (2009).

The electrical resistivities of all single crystalline samples and of one polycrystalline sample are shown in Figs.6b and 7 as function of temperature. While the resistivity of the 
polycrystalline sample drops to very low values at low temperatures, the residual resistivities of the single crystals are considerably higher. They increase in the sequence $p c \rightarrow s c 5 t \rightarrow$ $s c 1 t \rightarrow s c 5 b \rightarrow s c 1 b \rightarrow s c 2 \rightarrow s c 3$. This is about the same sequence in which the temperature and the sharpness of the low-temperature phase transition of the $C_{p} / T$ curves decreases (Fig. 6a). Thus, it is natural to assume that in the sequence $p c \rightarrow(s c 1 t$ and $s c 5) \rightarrow s c 1 b \rightarrow s c 2$ $\rightarrow s c 3$ the lattice disorder increases. Since the starting material purity of $s c 1 t$ and $s c 1 b$ was lower than that of $s c 2$, we conclude that the main reason of the disorder is a deviation of the sample composition from the exact stoichiometry 3:20:6 and not the concentration of foreign atoms. For single crystal sc4 the high temperature minimum occurs at lower temperatures than expected, leading to a slightly lower residual resistivity than for $s c 3$.

In $s c 1$ a remarkable increasing of the quality from the bottom to the top part of the crystal is observed. Only a small (top) part of the total crystal has an excellent quality and can be used for physical property investigations. $s c 5$ is indeed ranked after $s c 1 t$ according to the $C_{p}$ data but it is more homogeneous throughout the whole volume of the batch. A scenario which can explain the difference between the bottom $(s c 1 b)$ and the top $(s c 1 t)$ parts of the crystal $s c 1$ is as follows. At first (bottom part of the ingot) the crystal phase captures less Ce and more Pd from the stoichiometric melt (Table 1). While the crystal grows the melt gets enriched by Ce and depleted by Pd. At the end of the crystallisation (top part) this change in the melt composition results in a shift of the crystal composition to a more stoichiometric one, in accordance with the law of mass action. It was this observation that motivated us to perform the off-stoichiometric growth with a little Ce excess and Pd deficiency that resulted in sc5. As Fig. 7 shows the low temperature (down to $2 \mathrm{~K}$ ) relative resistivity of $s c 5$ is comparable with the best sample $s c 1 t$ and the spatial (top-bottom) difference in the resistivity is much smaller for $s c 5$ than for $s c 1$.

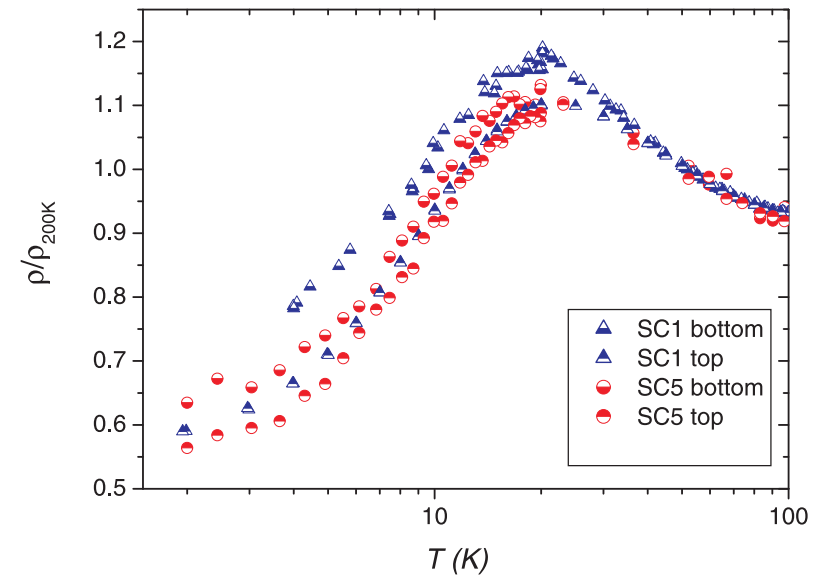

Fig. 7. Relative resistivities of the bottom and top parts of $s c 1$ and $s c 5$.

The highest residual resistance ratio $(R R R$, defined here as $\rho(200 \mathrm{~K}) / \rho(50 \mathrm{mK}))$ and the sharpest and most pronounced phase transition features in $C_{p} / T$ are found for the polycrystalline sample $(p c)$ which therefore appears to be the most stoichiometric one. This can be easily understood by the specifics of preparation. The accuracy of the stoichiometric total composition of a polycrystalline sample is limited only by the accuracy of the weighing process of starting materials and by their purity. A possible high temperature non-stoichiometry of the main phase of an as-cast polycrystal is compensated by the presence 
of minor impurity phases, the phase separation being heterogeneous on a microscopic scale. This heterogeneity can be lifted by annealing at lower temperatures due to the short diffusion path. During single crystal growth, however, a macroscopic phase separation can occur, making annealing very inefficient. Actually the resistivity curves of $s c 1 b$ before and after annealing were practically identical.

The Ce content $\Delta \mathrm{Ce}$ varies by more than 3 at.\% among all grown single crystals. We have revealed a systematic dependence of the residual resistance ratio, the lattice parameter, the (lower) phase transition temperature $T_{L}$, and the maximum in the temperature dependent electrical resistivity $T_{\max }$ with $\Delta$ Ce. This clarifies the sizable variation in the values of $T_{L}$ reported in the literature. We discuss the physical origin of the observed composition-property relationship in terms of a Kondo lattice picture. We predict that a modest pressure can suppress $T_{L}$ to zero and thus induce a quantum critical point.

While no clear correlation between the physical properties and the Si content can be demonstrated, a pronounced dependence on the Ce content (or anti-correlated Pd content) is observed. Figure 8a shows the dependencies of the lattice parameter $a$ and the residual resistance ratio $R R R$ as function of the deviation from stoichiometry $\Delta C$ e. The largest deviations from the "intrinsic behavior" (which is most closely met by the polycrystal) are seen for $s c 4$. It has the lowest Ce content of 7.4 at. $\%(\Delta \mathrm{Ce}=2.9$ at. \%). The polycrystalline sample $(p c)$ which demonstrates the most pronounced phase transition features and $s c 1 t$ with the second-sharpest features have the highest Ce contents, - and the lowest deviation from the exact Ce stoichiometry. The deviations $\Delta \mathrm{Ce}$ of $s c 1 t$ and sc5 lie on the other side of the stoichiometry line. One may argue that only the absolute value of $\Delta \mathrm{Ce}$ is relevant with respect to the composition-disorder-property relationship since any non-stoichiometry is usually associated with lattice imperfection. To test this conjecture we plot, in Fig. 8a, the data point for $s c 1 t$ also mirrored through the $\Delta \mathrm{Ce}=0$ axis (open symbol). Indeed this point fits nicely into the overall $R R R(\triangle \mathrm{Ce})$ behavior.

As it was pointed out in Sec. 2.4, the Ce-understoichiometry may be realized by two ways: either by Ce-vacancies or by Ce-vacancies partially (to 50\%) filled by Pd atoms. Both cases of Ce under-stoichiometry correspond, in a Kondo lattice description, to Kondo holes. In the Kondo coherent state at low temperatures Kondo holes act as strong scattering centers, decreasing the $R R R$. This effect is seen in Fig. 8a. Since also $s c 1 t$ has a reduced $R R R$, over-stoichiometry appears to be indeed realized by the combination of excess Ce and $\mathrm{Pd} / \mathrm{Si}$ vacancies. Excess Ce, just as Ce holes, creates Kondo disorder. In addition to reducing $R R R$, Kondo disorder is expected to successively suppress the temperature $T_{\max }$ where the high-temperature incoherent Kondo scattering with an approximate $\rho \propto-\ln T$ behavior crosses over to coherent Kondo scattering at low temperatures. In samples sc3 and sc4 Kondo disorder is so strong that $\rho \propto-\ln T$ is observed in a wide temperature range, and $\rho(T)$ shows only a tiny drop below $2 \mathrm{~K}$. Figures $6 \mathrm{~b}$ and 7 show that a sizable suppression of $T_{\max }$ occurs in our sample series $p c-(s c 1 t$ and $s c 5)-s c 1 b-s c 2-s c 3-s c 4$ only from $s c 2$ on. Thus, in addition to Kondo disorder, there appears to be a second effect influencing $T_{\max }$ in the opposite direction. This can be identified as a volume effect: the Ce vacancies or (smaller) $\mathrm{Pd}$ atoms on Ce sites in Ce under-stoichiometric samples as well as the Pd or Si vacancies in the over-stoichiometric sample sc1t lead to a shrinkage of the crystal lattice, which is seen in Table 2 and in Fig. 8a. The decrease in volume with decreasing Ce content corresponds to positive (chemical) pressure. Using the bulk modulus of the closely related compound $\mathrm{Ce}_{3} \mathrm{Pd}_{20} \mathrm{Ge}_{6}[\sim 137.5 \mathrm{GPa}$ at $150 \mathrm{~K}$; Nemoto et al (2003)] we can convert the lattice shrinkage in our off-stoichiometric single crystals into a hypothetical external pressure $p$. 


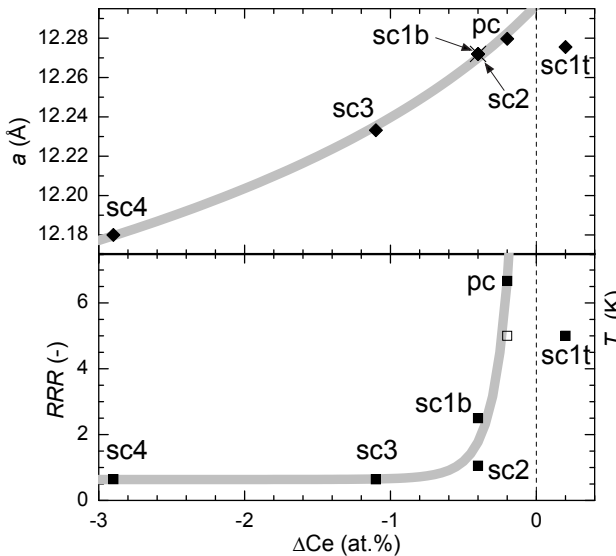

(a)

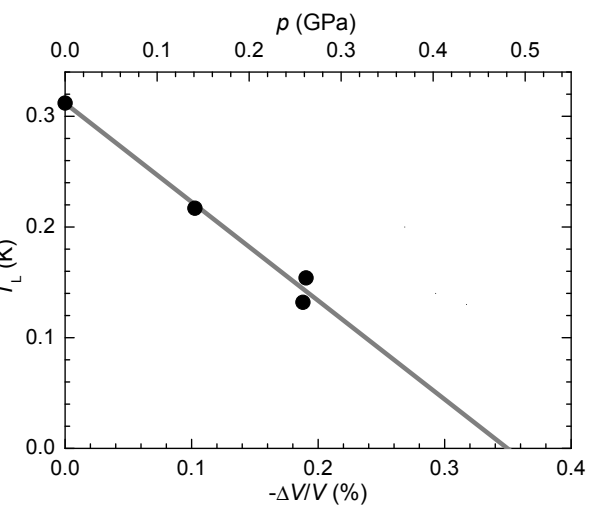

(b)

Fig. 8. a) Dependence of the lattice parameter $a$ and the residual resistance ratio $R R R$ on the deviation $\triangle \mathrm{Ce}$ from the stoichiometric Ce content. The open symbol in the lower panel represents the data point of $s c 1 t$, mirrored through the $\Delta \mathrm{Ce}=0$ line. The thick grey lines are guides to the eye. b) Lower transition temperature $T_{L}$ vs relative volume shrinkage $-\Delta V / V$ of the different $\mathrm{Ce}_{3} \mathrm{Pd}_{20} \mathrm{Si}_{6}$ single crystals with respect to the volume of the polycrystal $p c$. On the upper axis the corresponding pressure as estimated via the bulk modulus of $\mathrm{Ce}_{3} \mathrm{Pd}_{20} \mathrm{Ge}_{6}$ [Nemoto et al (2003)] is given. The line represents a linear fit to the data and its extrapolation to $T_{L}=0$. From Ref. Prokofiev et al (2009).

Figure 9 shows $T_{\max }$ (full dots) vs the relative volume shrinkage $-\Delta V / V$ of our single crystals with respect to our polycrystal $(p c)$ (bottom axis) and vs a hypothetical external pressure calculated via the bulk modulus [Nemoto et al (2003)] (top axis). The step-like, as opposed to continuous, decrease of $T_{\max }$ with $-\Delta V / V$, addressed already above, is clearly seen. In order to understand this behavior we compare our results to a pressure study [Hashiguchi et al (2000)]. We include the $T_{\max }$ vs $p$ data from this investigation on a polycrystalline sample under quasi-hydrostatic pressure in Fig. 9 (crosses and grey fit to these). From a value $T_{\max } \approx 20 \mathrm{~K}$ at $p=0$, similar to the value for our polycrystal, $T_{\max }$ increases continuously with increasing pressure. This behavior is typical for Ce-based heavy fermion compounds: with increasing pressure the hybridization between the $4 f$ electron of $\mathrm{Ce}$ and the conduction electrons increases and thus the Kondo temperature which is proportional to $T_{\max }$ increases. Our data follow this trend only at low non-stoichiometry (low $-\Delta V / V$ values) while at higher non-stoichiometry $T_{\max }$ decreases quickly. Thus we conclude that the volume effect on $T_{\max }$ dominates in our sample series $p c-s c 1 t-s c 1 b-s c 2-s c 3-s c 4$ up to $s c 1 b$ while the Kondo disorder effect dominates from $s c 2$ on.

Next we analyze the evolution of the ordering temperature $T_{L}$ along our sample series. For this purpose $T_{L}$, extracted from the specific heat data in Fig. 6a, is plotted vs $R R R$ in Fig. $9 \mathrm{~b}$ and vs $-\Delta V / V$ (bottom axis) or $p$ (top axis) in Fig. $8 \mathrm{~b} . T_{L}$ decreases continuously with decreasing $R R R$ (Fig. 9b). This might be taken as indication for the strong influence of disorder on $T_{L}$. However, in our sample series, the variation of $R R R$ is intimately linked to a variation of $\Delta \mathrm{Ce}$ (Fig. 8a) and thus to a variation of the lattice parameter $a$, the relative volume change $-\Delta V / V$, and the corresponding pressure $p$. Thus, an alternative 


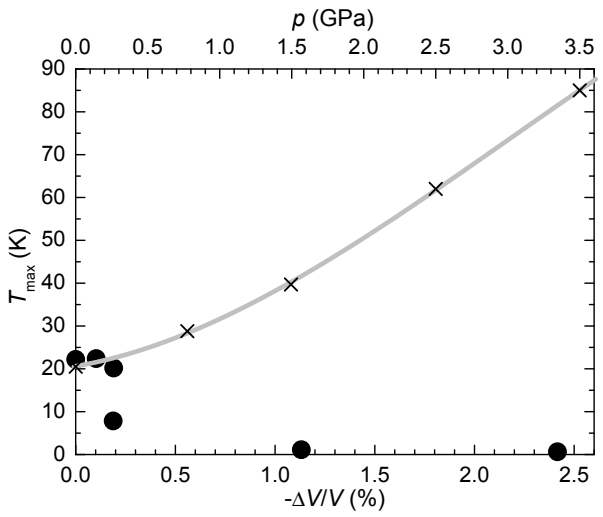

(a)

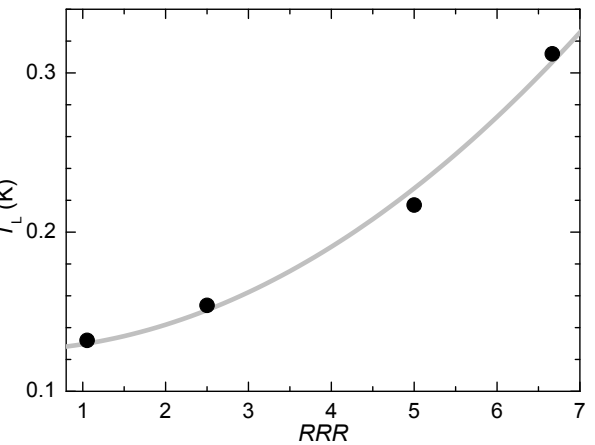

(b)

Fig. 9. a) Temperature $T_{\max }$ of the maximum in the electrical resistivity $v$ s relative volume shrinkage $-\Delta V / V$ of our $\mathrm{Ce}_{3} \mathrm{Pd}_{20} \mathrm{Si}_{6}$ single crystals with respect to the polycrystal (dots) and, for comparison, $T_{\max } v s$ pressure $p$ for the polycrystal of Hashiguchi et al (2000) (crosses and grey line, which is fit to the data). $\Delta V / V$ and $p$ are related to each other via the bulk modulus of $\mathrm{Ce}_{3} \mathrm{Pd}_{20} \mathrm{Ge}_{6} . \mathrm{b}$ ) Lower transition temperature $T_{L}$ vs residual resistance ratio $R R R$ for the different $\mathrm{Ce}_{3} \mathrm{Pd}_{20} \mathrm{Si}_{6}$ samples. The line represents a quadratic fit to the data. From Ref. Prokofiev et al (2009).

view of the situation is that not increasing disorder but decreasing volume is responsible for suppressing $T_{L}$ (Fig. 8b). To decide which effect is dominant we come back to our above discussion on Fig. $9 \mathrm{a}$ which revealed that the volume effect on $T_{\max }$ dominates in the series $p c-s c 1 t-s c 1 b-s c 2-s c 3-s c 4$ up to $s c 1 b$ while the disorder effect dominates from $s c 2$ on. If this holds true also for the effect on $T_{L}$ it is Fig. $8 \mathrm{~b}$ (without $s c 2$ ) that captures the essential physics while Fig. $9 \mathrm{~b}$ only displays implicit dependencies (except for $s c 2$ ).

In a Kondo lattice picture the physical origin of the suppression of $T_{L}$ with $p$ is that pressure increases the hybridization between conduction electrons and $4 f$ states, thus strengthening Kondo compensation, diminishing Ce magnetic moments, and suppressing the (most probably magnetic) ordering temperature $T_{L}$. Extrapolating the $T_{L}$ vs $p$ dependence of Fig. $8 \mathrm{~b}$ to higher $p$ suggests that a pressure induced quantum critical point is at reach for $\mathrm{Ce}_{3} \mathrm{Pd}_{20} \mathrm{Si}_{6}$. A linear extrapolation of the fit shown in Fig. $8 \mathrm{~b}$ yields $p_{c} \approx 0.5 \mathrm{GPa}$. Of course, actual low-temperature pressure measurements are needed to verify this possibility.

Finally, we comment on a related study on the germanide compound $\mathrm{Ce}_{3} \mathrm{Pd}_{20} \mathrm{Ge}_{6}$ where the influence of the starting composition on the physical properties was investigated [Kitagawa et al (1999)]. A strong composition effect on $C_{p}$ and $\rho$ was found to occur inside a narrow homogeneity range where a volume contraction of up to $0.7 \%$ takes place with increasing $\mathrm{Pd}$ content. In that work the concentration of $\mathrm{Pd}$ and not of $\mathrm{Ce}$ was concluded to be variable and responsible for the changing physical properties. It should be noted that the real composition of the resulting phases was not investigated. Thus, it is plausible that the increasing Pd content is accompanied by a decreasing Ce content. Under this assumption the composition effect on the properties of $\mathrm{Ce}_{3} \mathrm{Pd}_{20} \mathrm{Ge}_{6}$ and $\mathrm{Ce}_{3} \mathrm{Pd}_{20} \mathrm{Si}_{6}$ are indeed comparable. 


\section{3. $\mathrm{CeRu}_{4} \mathrm{Sn}_{6}$}

In Kondo insulators (frequently also referred to as heavy fermion semiconductors) a narrow gap develops at low temperatures in the electronic density of states at the Fermi level [Aepli \& Fisk (1992)]. While most Kondo insulators known to date adopt a cubic crystal structure (e.g., $\mathrm{YbB}_{12}, \mathrm{SmB}_{6}, \mathrm{FeSi}, \mathrm{Ce}_{3} \mathrm{Bi}_{4} \mathrm{Pt}_{3}$ ) a few compounds (e.g., CeNiSn, CeRhSb) are orthorhombic. These latter show anisotropic properties which suggest that the energy gap vanishes along certain directions in $k$-space. $\mathrm{CeRu}_{4} \mathrm{Sn}_{6}$, first synthesized by Das and Sampathkumaran [Das \& Sampathkumaran (1992)], crystallizes in a tetragonal structure of space group $I \overline{4} 2 m(a=6.8810 \AA, c=9.7520 \AA, c / a=1.4172)$ [Venturini et al (1990)]. A peculiarity of this compound is that, in addition to the tetragonal (body-centered) cell with lattice parameters $a$ and $c$ there exists a quasi cubic (face-centered) cell with lattice parameters $c^{\prime}$ and $c$, where $c^{\prime}$ is the diagonal of the tetragonal plane which differs by only $0.2 \%$ from $c$. This makes it very difficult to orient single crystals in an unambiguous way. On the other hand it allows us to study a "tetragonal" and a "quasi-cubic" Kondo insulator within the same material which is very appealing.

\subsection{Choice of the growth method and the growth procedure}

The compound $\mathrm{CeRu}_{4} \mathrm{Sn}_{6}$ melts incongruently. Melting followed by rapid quenching yields $\mathrm{CeRuSn}_{3}, \mathrm{Ru}_{3} \mathrm{Sn}_{7}$ and a tiny amount of the title phase in the solidified material. The incongruent melting was confirmed by a DTA experiment which showed multiple peaks on the heating and on the cooling curves (Fig. 10). Since crystal growth from the melt is impossible for an incongruently melting compound we searched for an appropriate flux. Usually low melting metals are used as high temperature solvents for intermetallic compounds. For $\mathrm{CeRu}_{4} \mathrm{Sn}_{6}$ this might be tin. However $\mathrm{CeRu}_{4} \mathrm{Sn}_{6}$ was shown to react with tin with the formation of other phases. To avoid possible contaminations by foreign elements we searched thereafter for a flux composition in the ternary Ce-Ru-Sn system (self-flux). Unfortunately, the Ce-Ru-Sn phase diagram which would be helpful for the choice of the best flux composition has not been reported. A Ce-rich flux for the Ce-poor $\mathrm{CeRu}_{4} \mathrm{Sn}_{6}$ is expected to lead to the formation of the highly stable phase CeRuSn ${ }_{3}$. Therefore, a Ce-free binary Ru-Sn mixture is more appropriate. Taking into account the Ru:Sn ratio of 2:3 in

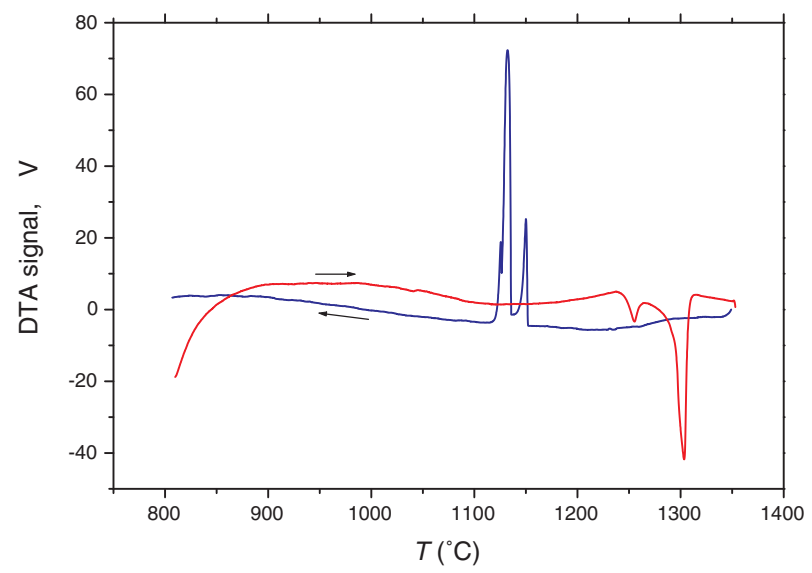

Fig. 10. DTA heating (red) and cooling (blue) curves of $\mathrm{CeRu}_{4} \mathrm{Sn}_{6}$. 
$\mathrm{CeRu}_{4} \mathrm{Sn}_{6}$ the flux composition " $\mathrm{Ru}_{2} \mathrm{Sn}_{3}$ " is optimal to maintain the Ru:Sn stoichiometry in the crystals. Moreover, the lowest melting $\left(1160^{\circ} \mathrm{C}\right)$ composition in the Ru-Sn binary diagram is the eutectic with the composition 42.5 at.\% Ru and 57.5 at.\% Sn [Massalski (1990)] which is close to the element ratio 2:3. According to Ananthasivan (2002) the system is a (partially immiscible) liquid above $1200^{\circ} \mathrm{C}$ in the composition range of $37-75$ at. $\%$ Sn. Finally, the large excess of $\mathrm{Ru}$ and $\mathrm{Sn}$ in the melt is expected to suppress the formation of the stable Ce-rich $\mathrm{CeRuSn}_{3}$ phase.

The crystals were grown by the floating-solution-zone traveling heater method (THM) in a mirror furnace. The seed and the feed rods had the stoichiometric composition whereas the melt zone was a solution of $\mathrm{CeRu}_{4} \mathrm{Sn}_{6}$ in $\mathrm{Ru}_{2} \mathrm{Sn}_{3}$. The growth rate was $0.3-1.0 \mathrm{~mm} / \mathrm{h}$. The composition of the crystals along their length in the growth direction was investigated by SEM/EDX analysis. No noticeable deviation from the stoichiometric ratio 1:4:6 can be observed (Fig. 11).

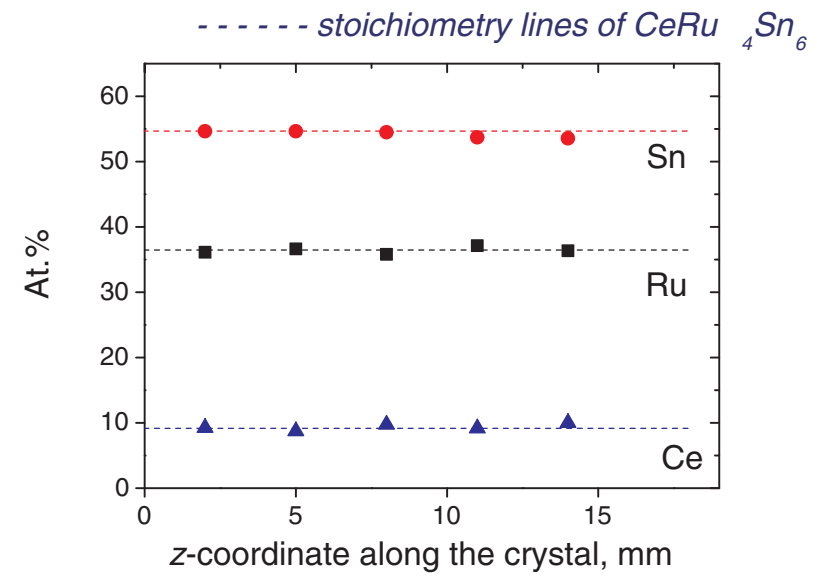

Fig. 11. Element concentration profiles of the $\mathrm{CeRu}_{4} \mathrm{Sn}_{6}$ single crystal in the growth direction.

\subsection{Physical properties of $\mathrm{CeRu}_{4} \mathrm{Sn}_{6}$ single crystals}

Figure 12 shows the temperature dependence of the electrical resistivity, $\rho(T)$, of $\mathrm{CeRu}_{4} \mathrm{Sn}_{6}$ on a semi-logarithmic scale. With decreasing temperature $\rho$ first increases steeply (range 1 ), then passes over a maximum at about $10 \mathrm{~K}$, increases again, albeit less steeply (range 2), and finally tends to saturate at the lowest temperatures. A possible explanation of this behaviour is a double-gap structure frequently encountered in simple semiconductors: a larger intrinsic gap visible at high temperatures (range 1) and a smaller extrinsic gap between impurity states and the band edge that dominates the low-temperature behaviour (range 2). Approximating $\rho(T)$ between 120 and $300 \mathrm{~K}$ with exponential behaviour $\left(\rho=\rho_{0} \exp \left(\Delta_{1} / 2 k_{B} T\right)\right.$, Arrhenius law) yields an energy gap $\Delta_{1} / k_{B}=125 \mathrm{~K}$, sizeably larger than previously reported for polycrystalline samples [Brünig et al (2006); Das \& Sampathkumaran (1992)]. Fitting the data between 0.8 and $1.8 \mathrm{~K}$ with the same function yields $\Delta_{2} / k_{B}=0.1 \mathrm{~K}$ (see full red lines in Fig. 12 for both fits). While this gap value may seem incongruous with respect to the fit range, it has to be bourn in mind that the influence of the low- $T$ gap on $\rho(T)$ is expected to diminish by eventual thermal depopulation of the upper states towards $T=0$ and hence the observed 
saturation in $\rho(T \rightarrow 0)$. Thus, a more complete fitting function is needed to account for these effects.

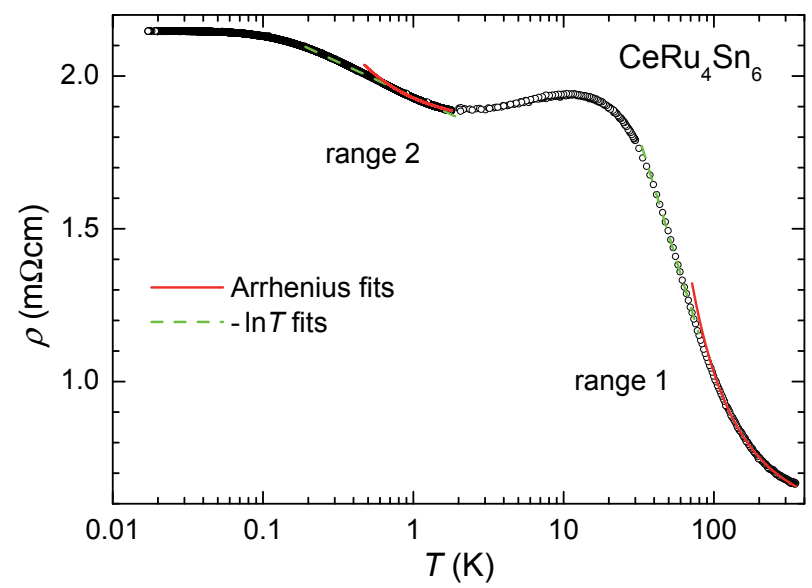

Fig. 12. Temperature dependence of the electrical resistivity, $\rho(T)$, of the $\mathrm{CeRu}_{4} \mathrm{Sn}_{6}$ single crystal on a semi-logarithmic scale. From Ref. Paschen et al (2010).

Now, having single crystals an investigation of the anisotropy of $\mathrm{CeRu}_{4} \mathrm{Sn}_{6}$ became feasible. The magnetic susceptibility was measured on an oriented single crystal in two mutually perpendicular directions (Fig. 13a). One of these directions is the crystallographic $c$ axis, the other one is situated within the tetragonal plane. A pronounced difference is seen. For both directions Curie Weiss-type behaviour is observed at high temperatures, with an effective magnetic moment that is roughly consistent with the full effective moment of $\mathrm{Ce}^{3+}$, and with the paramagnetic Weiss temperatures $\Theta_{\|_{c}} \approx 395 \mathrm{~K}$ and $\Theta_{\perp_{c}} \approx 155 \mathrm{~K}$ for $\mathrm{H} \| c$ and $\mathrm{H} \perp c$, respectively.

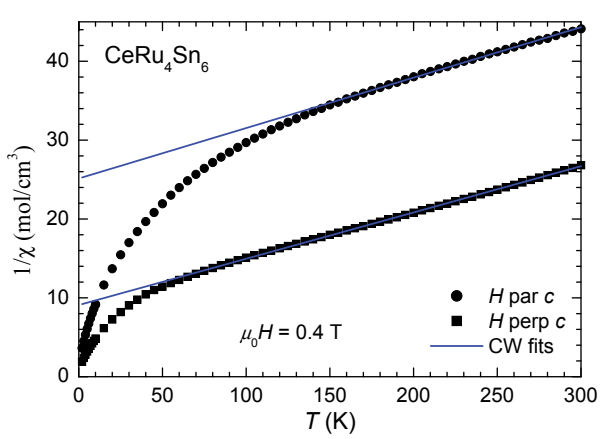

(a)

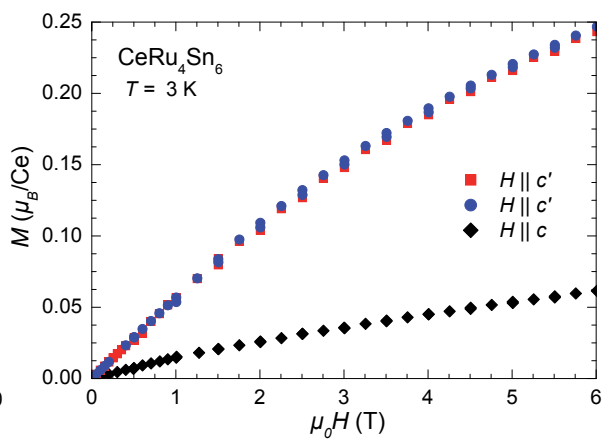

(b)

Fig. 13. a) Temperature dependence of the inverse magnetic susceptibility, $\chi^{-1}(T)$, of $\mathrm{CeRu}_{4} \mathrm{Sn}_{6}$ for the magnetic field $\mu_{0} H=0.4 \mathrm{~T}$ applied along the crystallographic $c$ axis and within the tetragonal plane. b) Magnetic field dependence of the magnetization, $M\left(\mu_{0} H\right)$, taken at $3 \mathrm{~K}$ for fields applied along the crystallographic $c$ and $c^{\prime}$ axes. Fig. 13b from Ref. Paschen et al (2010). 
In order to test whether anisotropy also exists within the quasi-cubic cell we prepared small single crystalline platelets (with geometries which allowed for specific heat measurements only) cut from one piece in such a way that three mutually perpendicular directions were obtained: for two samples a $c^{\prime}$ axis is perpendicular to the platelet plane, for one sample it is $c$. As explained above, our Laue diffractograms cannot identify which sample is which. Our specific heat measurements, however, allow to clearly identify the $c$ - and $c^{\prime}$-oriented samples: while the zero field data are very similar for all three samples a magnetic field applied perpendicular to the platelet planes induces sizable anisotropy. The difference is best seen by plotting the relative difference in specific heat induced by a magnetic field, $\left(c_{p}(B)-c_{p}(0)\right) / c_{p}(0)$, which reaches a maximum of more than $70 \%$ at $3.5 \mathrm{~K}$ for $B \| c^{\prime}$ but is below $15 \%$ for $B \| c$ at this temperature (Fig. 14). Two samples (sc1 and $s c 2$ ) show very similar behavior and must thus be $c^{\prime}$-oriented while sc 3 shows distinctly different behavior and is thus identified as the $c$-oriented sample.

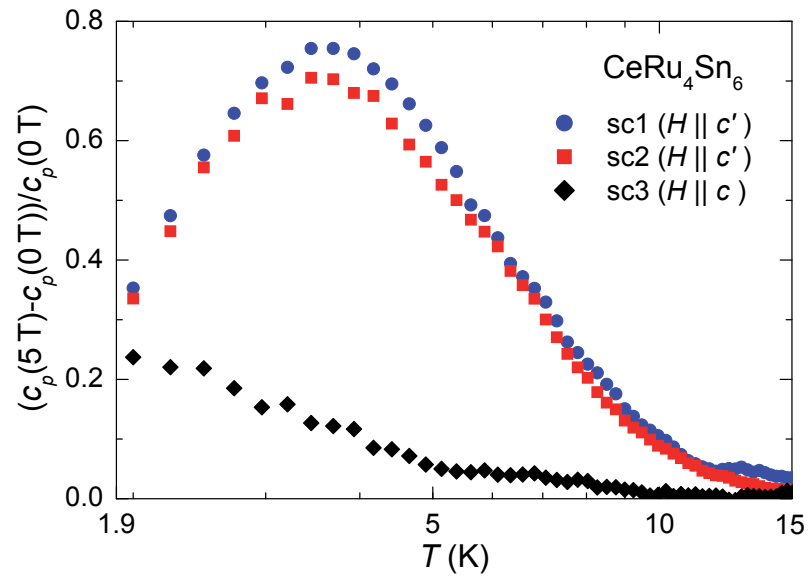

Fig. 14. Temperature dependence of the surplus specific heat induced by a magnetic field of 5 $\mathrm{T},\left(c_{p}(5 \mathrm{~T})-\mathrm{c}_{p}(0)\right) / \mathrm{c}_{p}(0)$, of the three $\mathrm{CeRu}_{4} \mathrm{Sn}_{6}$ single crystals $s c 1, s c 2$, and $s c 3$. From Ref.

Paschen et al (2010).

A possible interpretation of these data is that a narrow energy gap which is present along $c^{\prime}$ in zero field is suppressed/diminished by a field of $5 \mathrm{~T}$ in this direction. Since the same field applied along $c$ leads to a much weaker increase of $c_{p}$ we believe that no or a much less field sensitive gap is present along $c$ [Paschen et al (2010)].

\section{CeAuGe}

A sizable number of $\mathrm{Ce}$ - and $\mathrm{Yb}$-based intermetallic compounds demonstrate quantum critical behaviour. Most of the compounds are antiferromagnets, whereas only a handful of Ce and $\mathrm{Yb}$ compounds with a ferromagnetic phase transition at low temperatures is known. The occurrence of quantum criticality in a ferromagnetic ground state is a subject of current debate. CeAuGe orders ferromagnetically at a relatively low Curie temperature of $T_{C}=10.9 \mathrm{~K}$ [Sondezi-Mhlungu et al (2009), Mhlungu \& Strydom (2008)]. Thus it can be expected that the magnetic order can be tuned or fully suppressed by modest variations in magnetic field or pressure. 
CeAuGe is a phase with a homogeneity range. Stoichiometric and nearly stoichiometric $\mathrm{CeAuGe}$ adopts the NdPdSb structure, an ordered variant of the $\mathrm{AlB}_{2}$ structure type. The unit cell is hexagonal (space group $P 6_{3} m c$ ) with the lattice parameters $a=4.4569 \AA$ and $c=$ $7.9105 \AA$ [Pöttgen et al (1998)]. At larger deviations from the elemental 1:1:1 ratio a phase with a slightly different structure forms. The non-stoichiometric $\mathrm{CeAu}_{1-x} \mathrm{Ge}_{1+x}$ crystallizes in the true $\mathrm{AlB}_{2}$ structure type and has about twice smaller unit cell. Unlike the ordered 1:1:1 phase, $\mathrm{Au}$ and $\mathrm{Ge}$ atoms are distributed statistically and the $\mathrm{Au} / \mathrm{Ge}$ layers are not puckered but planar [Jones et al (1997)].

Since CeAuGe is not cubic it is especially important to investigate its physical properties on oriented single crystalline samples. We have investigated the crystal growth of this phase of various stoichiometries by the floating zone technique. In the course of the growth experiments we encountered a severe non-stoichiometry problem. We report here on our efforts to diminish the deviation from the 1:1:1 stoichiometry and the segregation effects resulting from it.

\subsection{Crystal growth using stoichiometric feed rods}

As a starting point, growth from the stoichiometric melt was tried. We studied the evolution of the crystal composition during the crystallization by measuring the composition at the starting, middle and final part of the crystallized ingot by the EDX technique. The concentration profiles are represented in Figs. 15 and 16. At the left border of each panel the initial compositions of the respective polycrystalline feed rod is shown. The beginning of the crystallization corresponds thus to the length coordinate $z=0$. As Fig. 15a shows, the crystals primarily crystallized from the stoichiometric melt (i.e. the first portion of the crystalline phase) have a non-stoichiometric composition with a reduced Au content and increased $\mathrm{Ce}$ and Ge contents. This leads to a change of the melt composition with an accumulation of $\mathrm{Au}$ and a depletion of $\mathrm{Ce}$ and $\mathrm{Ge}$. As a consequence, the Au content increases in the crystals and the Ce and Ge contents decrease in the course of further crystallization (Fig. 15a).

Due to the composition change the lattice parameters change too (Fig. 15b). For the first solidified crystals the a-parameter is lower and the c-parameter is higher than the stoichiometric values (Fig. 15b). Further crystallization leads to a decrease of $c$ and to an increase of $a$.

Thus, the growth using a stoichiometric feed rod results in a non-stoichiometric single crystalline ingot which, along its length, is macro-inhomogeneous with respect to all three constituting elements.

\subsection{Crystal growth using off-stoichiometric feed rods}

In order to suppress the above discussed starting deviation in the element concentration in the crystals with respect to the melt we used a feed rod enriched in Au and depleted in Ge content, with the off-stoichiometric composition $\mathrm{CeAu}_{0.96} \mathrm{Ge}_{1.04}$. The primary crystals obtained from this run appear to be nearly stoichiometric (Fig. 16a, $\mathrm{z}=0 \mathrm{~mm}$ ), but in the course of further crystallization the composition again drifts away from the 1:1:1 stoichiometry: the Au content increases and the Ge content decreases, both summing up to a constant value. However, the Ce content remains constant along the whole solidified ingot (Fig. 16a). This fact is favorable for physical investigations on large crystals because non-stoichiometry of Ce is usually the most disturbing factor in heavy fermion systems. The crucial role of the Ce stoichiometry was demonstrated in Section 2 on $\mathrm{Ce}_{3} \mathrm{Pd}_{20} \mathrm{Si}_{6}$. Crystallization from the off-stoichiometric melt $\mathrm{CeAu}_{0.96} \mathrm{Ge}_{1.04}$ seems to be most promising for the growth of stoichiometric homogeneous 


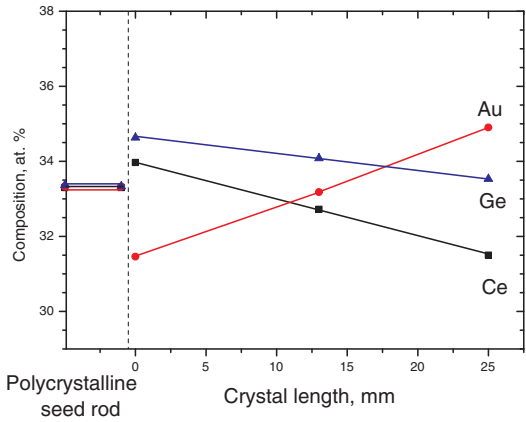

(a)

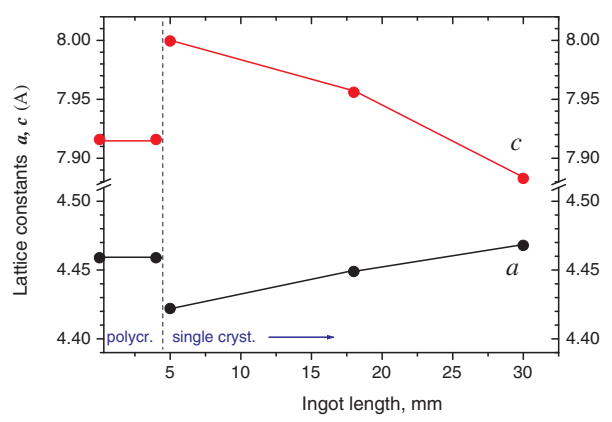

(b)

Fig. 15. a) Element concentration profiles along the growth direction at the crystallization from a stoichiometric feed rod. b) Profiles of the unit cell parameters $a$ and $c$.

CeAuGe crystals, provided that the growth technique should be modified. In the floating zone method the melt-to-crystal volume ratio is very small. Therefore the segregation phenomena have very strong impact on the composition of the melt zone, and the crystal composition varies strongly during the growth. In other techniques, e.g. the Czochralski method, the melt-to-crystal volume ratio can be rather large, and the growth of homogeneous (also with respect to the $\mathrm{Au} / \mathrm{Ge}$ ratio) crystals from the melt with a practically constant composition appears feasible.

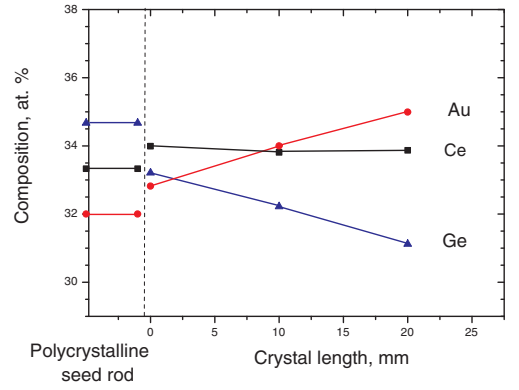

(a)

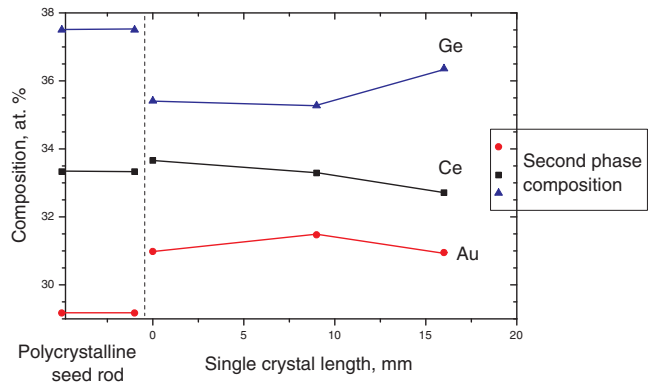

(b)

Fig. 16. Element concentration profiles along the growth direction for crystal growth from off-stoichiometric feed rods with the compositions (a) $\mathrm{CeAu}_{0.96} \mathrm{Ge}_{1.04}$ and (b) $\mathrm{CeAu}_{0.88} \mathrm{Ge}_{1.12}$.

Crystallization from a more strongly off-stoichiometric melt $\mathrm{CeAu}_{0.88} \mathrm{Ge}_{1.12}$ yielded strongly non-stoichiometric crystals with a large excess of $\mathrm{Ge}$ and a lack of $\mathrm{Au}$, the $\mathrm{Ce}$ content remaining at nearly stoichiometric level (Fig. 16b.)

While the primarily crystallized material is, according to SEM, single phased, the finally solidified ingot consists of two phases (Fig. 17, right). These two phases are the ordered and the disordered variants of the $\mathrm{AlB}_{2}$ structure (the $\mathrm{NdPdSb}$ and the true $\mathrm{AlB}_{2}$ types). The material from the middle part of the ingot seems to be single-phased at first glance (Fig. 17, upper left), but a closer inspection under higher magnification reveals a two-phase pattern (Fig. 17, lower left). The fine grained microstructure of the material (especially apparent in 
comparison with that of the final part (Fig. 17, right)) evidences for crystallisation of a single phase at first which was decaying into two phases later in the course of cooling. This behavior is the sign of a strong temperature dependence of the homogeneity range of the NdPdSb-type phase.

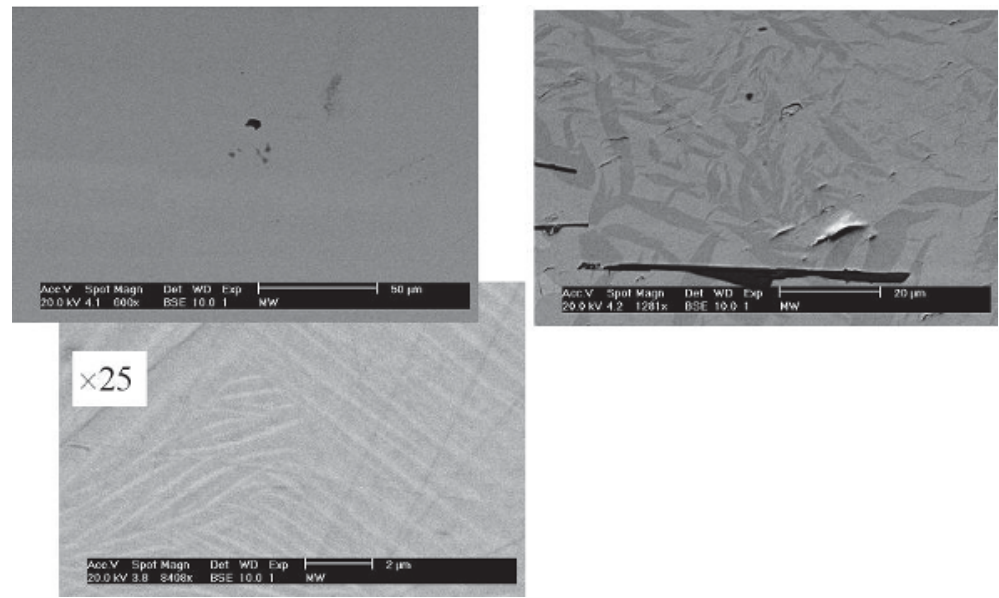

Fig. 17. Microstructure of the ingot crystallized from the feed rod with the composition $\mathrm{CeAu}_{0.88} \mathrm{Ge}_{1.12}$. Left - middle part, right - upper part. The magnified $(\times 25)$ image of the middle part shows a fine inhomogeneity of the material.

\section{Conclusions}

Single crystals of the $\mathrm{Ce}_{3} \mathrm{Pd}_{20} \mathrm{Si}_{6}$ phase were grown from the melt (stoichiometric and slightly off-stoichiometric) under various growth conditions and from high-temperature solutions using $\mathrm{Pd}_{5} \mathrm{Si}$ as a flux. $\mathrm{Ce}_{3} \mathrm{Pd}_{20} \mathrm{Si}_{6}$ melts quasi-congruently, i.e., the peritectic temperature is very close to the temperature of the complete melting. This fact follows from our DTA experiments and the observation of the melting zone during the growth process. The floating zone with stoichiometric composition was very unstable because of the low surface tension, which made the melt growth problematic. In contrast, the off-stoichiometric flux growth ran stably but resulted in non-stoichiometric single crystals.

While the Si content varies only slightly for different crystals, the Ce and Pd contents do so sizably, the decrease of Ce being partially compensated by an increase in $\mathrm{Pd}$. The existence of a homogeneity range in $\mathrm{Ce}_{3} \mathrm{Pd}_{20} \mathrm{Si}_{6}$ is the reason for the strong variation of the properties of single crystals grown by different techniques. The sharpness of the lower (presumably antiferromagnetic) phase transition, its transition temperature $T_{L}$, the residual resistivity, and the temperature $T_{\max }$ of the (local) maximum in $\rho(T)$ were shown to be measures of the crystal quality. Based on all these properties the upper part of the crystal grown from the stoichiometric melt $(s c 1 t)$ and the whole volume of the crystal sc5 grown from a slightly off-stoichiometric melt can be ranked as having the highest perfection among all the grown single crystals. Their lattice parameters together with their compositions indicate that the slight off-stoichiometry is not dominated by $\mathrm{Ce}$ on the $\mathrm{Pd}$ sites but by $\mathrm{Pd}$ vacancies, which do not directly disturb the $4 f$ lattice. 
$\mathrm{CeRu}_{4} \mathrm{Sn}_{6}$ melts incongruently. Single crystals of $\mathrm{CeRu}_{4} \mathrm{Sn}_{6}$ can be grown from $\mathrm{Ru}_{2} \mathrm{Sn}_{3}$ flux. The grown single crystals show no marked deviation from the ideal stoichiometry, which indicates a very narrow homogeneity range of the phase. On the grown crystals anisotropies of the magnetic properties of two types were demonstrated: along the tetragonal unit cell axes and along the axes of a quasi-cubic unit cell.

Single crystal growth of CeAuGe is complicated by a wide homogeneity range of the phase. Growth from the stoichiometric melt yields single crystals with an essential deviation from the stoichiometry. The composition of single crystals varies strongly along the growth direction. The crystal composition depends complexly on the melt composition and on the crystallisation temperature (the latter is a function of the former). Growth from the off-stoichiometric melt $\mathrm{CeAu}_{0.96} \mathrm{Ge}_{1.04}$ is optimal for nearly stoichiometric crystals provided that the melt-to-crystal volume ratio is large enough for keeping the melt composition quasi-constant during the entire growth run.

\section{Acknowledgement}

We are grateful to A. Strydom and co-workers for feed rod preparation of the CeAuGe phases, to K. Neumaier for $C_{p}$ measurements on $s c 5$, and to $\mathrm{H}$. Ossmer for resistivity measurements on sc5. We thank M. Waas and V. Peter for the SEM/EDX measurements and for metallographic sample preparation. The work was financially supported by the Austrian Science Foundation (project P19458-N16) and the European Research Council (Advanced Grant n ${ }^{\circ} 227378$ ).

\section{References}

Aeppli G. and Fisk Z., Comments Condens. Matter Phys. 16, 155 (1992).

Ananthasivan K., Kaliappan I., Vasudeva Rao P.R., Sudha C., Terrance A.L.E.. J. Nucl. Mater., 305, 97-105 (2002)

Brünig E. M., Baenitz M., Gippius A. A., Paschen S., Strydom A. M., and Steglich F., Physica B 378Ü380, 839 (2006).

Chandrasekharaiah M., in Binary Alloy Phase Diagrams, 2nd ed., edited by T. Massalski (William W.Scott, Jr., USA, 1990).

Das I. and Sampathkumaran E. V., Phys. Rev. B 46, 4250 (1992).

Dung N., Haga Y., Matsuda T., Yamada T., Thamizhavel A., Okuda Y., Takeuchi T., Sugiyama K., Hagiwara M., Kindo K., Settai R., and Onuki Y., J. Phys. Soc. Jpn. 76, 024702 (2007).

Goto T., Watanabe T., Tsuduku S., Kobayashi H., Nemoto Y., Yanagisawa T., M.Akatsu, Ano G., Suzuki O., Takeda N., Dönni A., and Kitazawa H., J. Phys. Soc. Jpn. 78, 024716 (2009).

Gribanov A. V., Seropegin Y. D., and Bodak O. J., J. Alloys Compd. 204, L9 (1994).

Gribanov A. V., Rogl P., and Seropegin Y. D., in Noble metal systems, in Landolt-Börnstein, New Series (Springer, Berlin, 2006), Vol. IV/11B, p. 340.

Hashiguchi T., Takeda N., Kitagawa J., Wada N., Takayanagi S., Ishikawa M., and Mori N., J. Phys. Soc. Jpn. 69, 667 (2000).

Jones C.D.W., Gordon R.A., DiSalvo F.J., Pöttgen R., Kremer R.K. J. Alloys \& Comp. 260, 50 (1997)

Kitagawa J., Takeda N., and Ishikawa M., Phys. Rev. B 53, 5101 (1996).

Kitagawa J., Takeda N., Ishikawa M., Yoshida T., Ishiguro A., Kimura N., and T.Komatsubara, Phys. Rev. B 57, 7450 (1998). 
Kitagawa J., Takeda N., Sakai F., and Ishikawa M., J. Phys. Soc. Jpn. 68, 3413 (1999).

In: Binary alloy phase diagrams, T.B. Massalski, (Ed.), V. 3, 3255, William W. Scott, Jr., ISBN 0-87170-406-4.

Mhlungu B.M., Strydom A.M.. Physica B 403, 862 (2008)

Mitamura H., Tayama T., Sakakibara T., Tsuduku S., Ano G., Ishii I., Akatsu M., Nemoto Y., Goto T., Kikkawa A., Kitazawa H., J. Phys. Soc. Jpn. 79, 074712 (2010).

Nemoto Y., Yamaguchi T., Horino T., Akatsu M., Yanagisawa T., Goto T., O.Suzuki, Dönni A., and Komatsubara T., Phys. Rev. B 68, 184109 (2003).

Paschen S., in Thermoelectrics Handbook (ed. D. M. Rowe, CRC Press, Boca Raton, 2006), Chap. 15 (Thermoelectric aspects of strongly correlated electron systems).

Paschen S., Müller M., Custers J., Kriegisch M., Prokofiev A., Hilscher G., Steiner W., Pikul A., Steglich F., and Strydom A. M., J. Magn. Magn. Mater. 316, 90 (2007).

Paschen S., Laumann S., Prokofiev A., Strydom A. M., Deen P. P., Stewart J. R., Neumaier K., Goukassov A., and Mignot J.-M., Physica B 403, 1306 (2008).

Paschen S., Winkler H., Nezu T., Kriegisch M., Hilscher G., Custers J., Prokofiev A., Strydom A. J. Phys.: Conf. Ser. 200, 012156 (2010)

Pöttgen R., Bormann H., Kremer R.K., J. Magn. Magn. Mater. 152, 196 (1996)

Prokofiev A., Custers J., Kriegisch M., Laumann S., Müller M., Sassik H., Svagera R., Waas M., Neumaier K., Strydom A. M., Paschen S., Phys. Rev. B 80, 235107 (2009).

Seropegin Y. D., Gribanov A. V., Kubarev O. L., Tursina A. I., and Bodak O. I., J. Alloys Comp. 317-318, 320 (2001).

Sondezi-Mhlungu B.M., Adroja D.T., Strydom A.M., Paschen S., Goremychkin E.A.. Physica B 404, 3032 (2009)

Strydom A. M., Pikul A., Steglich F., and Paschen S., J. Phys.: Conf. Series 51, 239 (2006).

Takeda N., Kitagawa J., and Ishikawa M., J. Phys. Soc. Jpn. 64, 387 (1995).

Venturini G., Chafik El Idrissi B., Marêché J., and Malaman B., Mater. Res. Bull. 25, 1541 (1990). 


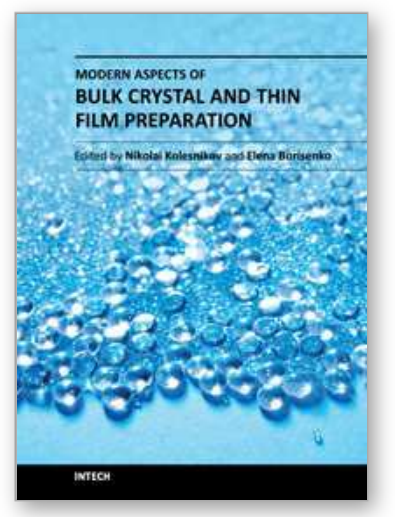

\author{
Modern Aspects of Bulk Crystal and Thin Film Preparation \\ Edited by Dr. Nikolai Kolesnikov
}

ISBN 978-953-307-610-2

Hard cover, 608 pages

Publisher InTech

Published online 13, January, 2012

Published in print edition January, 2012

In modern research and development, materials manufacturing crystal growth is known as a way to solve a wide range of technological tasks in the fabrication of materials with preset properties. This book allows a reader to gain insight into selected aspects of the field, including growth of bulk inorganic crystals, preparation of thin films, low-dimensional structures, crystallization of proteins, and other organic compounds.

\title{
How to reference
}

In order to correctly reference this scholarly work, feel free to copy and paste the following:

Andrey Prokofiev and Silke Paschen (2012). Crystal Growth and Stoichiometry of Strongly Correlated Intermetallic Cerium Compounds, Modern Aspects of Bulk Crystal and Thin Film Preparation, Dr. Nikolai Kolesnikov (Ed.), ISBN: 978-953-307-610-2, InTech, Available from:

http://www.intechopen.com/books/modern-aspects-of-bulk-crystal-and-thin-film-preparation/crystal-growthand-stoichiometry-of-strongly-correlated-intermetallic-cerium-compounds

\section{INTECH}

open science | open minds

\section{InTech Europe}

University Campus STeP Ri

Slavka Krautzeka 83/A

51000 Rijeka, Croatia

Phone: +385 (51) 770447

Fax: +385 (51) 686166

www.intechopen.com

\section{InTech China}

Unit 405, Office Block, Hotel Equatorial Shanghai

No.65, Yan An Road (West), Shanghai, 200040, China 中国上海市延安西路65号上海国际贵都大饭店办公楼 405 单元

Phone: +86-21-62489820

Fax: $+86-21-62489821$ 
(C) 2012 The Author(s). Licensee IntechOpen. This is an open access article distributed under the terms of the Creative Commons Attribution 3.0 License, which permits unrestricted use, distribution, and reproduction in any medium, provided the original work is properly cited. 